\title{
Bedside lung ultrasound guided the treatment of neonatal respiratory distress syndrome by mechanical ventilation
}

\author{
Zhao Chun*, Cheng Yingying, Xu Linger, Fu Huihui and Yuan Yebo \\ Department of Paediatrics, Yuyao People's Hospital, The Affiliated Yangming Hospital of Ningbo University, China
}

\begin{abstract}
Neonatal respiratory distress syndrome is one of the leading causes of neonatal death. Lung ultrasound has been used in the diagnosis of neonatal pulmonary diseases as a new non-invasive examination method in recent years. In this study, ten infants with neonatal respiratory distress syndrome received mechanical ventilation underwent lung ultrasound examination. The results show that there were significant differences in lung ultrasound scores before and after mechanical ventilation treatment. But two of the cases failed at the first disconnection. In conclusion, lung ultrasound has certain application value and specific advantages in guiding NRDS mechanical ventilation treatment. A comprehensive understanding of lung ultrasound and further standardization of its operation and evaluation criteria will enable its further development and popularization.
\end{abstract}

\section{Introduction}

Neonatal respiratory distress syndrome (NRDS) is caused by the lack of pulmonary surfactant and immature pulmonary structure due to immature alveolar function. It is common in premature infants with gestational age less than 35 weeks. It can also occur in new-borns of diabetic mothers and caesarean babies. Of course, SP-A gene mutation and SP-B gene defect will cause pulmonary surfactant to fail to play its due role in perinatal asphyxia and neonates with severe $\mathrm{Rh}$ haemolytic disease. The main clinical manifestations were progressive dyspnoea, cyanosis and respiratory failure within a few hours after birth [1]. At present, the treatment of NRDS is becoming more and more mature, and the survival rate of premature infants at low gestational age is also increasing. However, some complications, such as bronchopulmonary dysplasia, have not been further controlled. Therefore, the early diagnosis and follow-up of NRDS have a significant impact on children's long-term quality of life.

At present, NRDS is a disease with high perinatal morbidity and mortality in both developed and developing countries. The incidence of NRDS in 28-week-old premature infants was $80 \%$, while that in 24 -week-old premature infants was $95 \%$ [2]. The incidence of NRDS in China is slightly lower than that in European and American countries. The incidence rate was $60 \% \sim 80 \%$ when the gestational age was less than 28 weeks, $15 \% \sim 30 \%$ when the gestational age was $32 \sim 36$ weeks, and $5 \%$ when the gestational age was more than 37 weeks [3]. Therefore, the diagnosis and treatment of RDS is of great significance to improve the overall survival rate of new-borns.

Ultrasound has become the preferred diagnostic tool for many clinical diseases because of its non-invasive, immediate, convenient, fast and non-radiation advantages. However, because the alveoli are full of air, ultrasound can completely reflect when it meets the air, so lung ultrasound has not been a routine auxiliary examination of the chest, and the diagnosis of lung diseases has long relied on chest X-ray examination. With the increasing demand of critical care medicine and the popularity of bedside ultrasound, the study of lung ultrasound has been paid more and more attention. Existing studies have found that ultrasound has high sensitivity and specificity in the diagnosis of pulmonary diseases, and has been used in the diagnosis of a variety of pulmonary diseases in adults and children [4-11].

At present, lung ultrasound has been applied in the field of emergency and critical medicine for adults and children. In 2012, experts of the International Federation of Lung Ultrasound have reached an international consensus that lung ultrasound can accurately diagnose community-acquired pneumonia, acute respiratory distress syndrome, atelectasis, pulmonary oedema and pneumothorax, and even some pulmonary diseases. The diagnostic accuracy and sensitivity of lung ultrasonography are higher than those of chest X-ray [7-10,1214]. And lung ultrasound is more suitable for application in intensive care units and neonatal care units because of its unique advantages [15-17]. In this study, bedside lung ultrasound was used to evaluate the characteristics of lung ultrasonography in infants with NRDS, and to dynamically monitor the measurements before and after mechanical ventilation in infants with NRDS, so as to provide clinical guidance for the clinical diagnosis and evaluation of curative effect of RDS.

\section{Method}

10 neonates who were clinically diagnosed with neonatal respiratory distress syndrome by blood gas analysis and chest X-ray examination and treated with mechanical ventilation from July 2018 to January 2019 in our hospital were selected as the observation group, including 5 males and 5 females, with gestational age $(27+6)$ weeks

*Correspondence to: Zhao Chun, Department of Paediatrics, Yuyao People's Hospital, The Affiliated Yangming Hospital of Ningbo University, Chengdong Road 800, Ningbo Zhejiang, China, Tel: 0086 15267852005; E-mail: yyzhaochun@163.com

Key words: bedside lung ultrasound, neonatal respiratory distress syndrome, mechanical ventilation

Received: September 05, 2019; Accepted: September 23, 2019; Published: September 27, 2019 
to $(32+2)$ weeks, and birth weight ranging from $0.9 \mathrm{~kg}$ to $1.7 \mathrm{~kg}$. Ultrasonic examination was performed by using Mindray M7 Expert ultrasonic diagnostic instrument. Linear array probes were selected, and the frequency was set at $10 \sim 11 \mathrm{MHz}$. In a quiet state, neonates were placed in supine, lateral, and prone positions. Each lung was divided into anterior, lateral, and posterior regions with the anterior axillary line and posterior axillary line. The two nipples were connected as boundaries, and each lung was divided into two upper and lower lung fields, so that the two lungs were divided into 12 regions. The probe shall conduct transverse and longitudinal scanning of the lung starting from the second intercostal area. During longitudinal scanning, the probe should be perpendicular to the ribs. During transverse scanning, the probe should conduct intercostal scanning one by one along the intercostal space $[18,19]$. The observation indexes include pleural line, A-line, B-line, pulmonary interstitial syndrome, white lung, pulmonary consolidation, double lung point. Lung ultrasound score: 0: Mainly A line, less than two isolated B line, pulmonary sliding sign can be seen. One point: Multiple B lines with clear boundaries, regular or irregular distribution and spacing greater than $7 \mathrm{~mm}$; Two points: Dense B line with diffuse distribution and spacing less than $3 \mathrm{~mm}$, with or without waterfall sign; Three points: Lung consolidation [20,21]. SPSS 16.0 software was used for data analysis.

\section{Results}

The mean lung ultrasound score of 10 infants with NRDS before mechanical ventilation was $1(31.40 \pm 4.03)$ point, and the mean lung ultrasound score of 10 infants with NRDS after successful removal was $(13.20 \pm 3.29)$ points, the difference between them was statistically significant $(P<0.01$, Figure 1$)$. Two of the infants showed respiratory distress again within 12 hours after the first withdrawal, and the lung ultrasound imaging manifestations were monitored to deteriorate (Figure 2). The ultrasound scores were increased, and the infants were given auxiliary ventilation again.

\section{Discussion}

Findings from this study confirmed that lung ultrasound can be used to guide mechanical ventilation in the treatment of neonatal respiratory distress syndrome. Wang conducted bedside ultrasound scores on 49 children with NRDS, and the results showed that lung

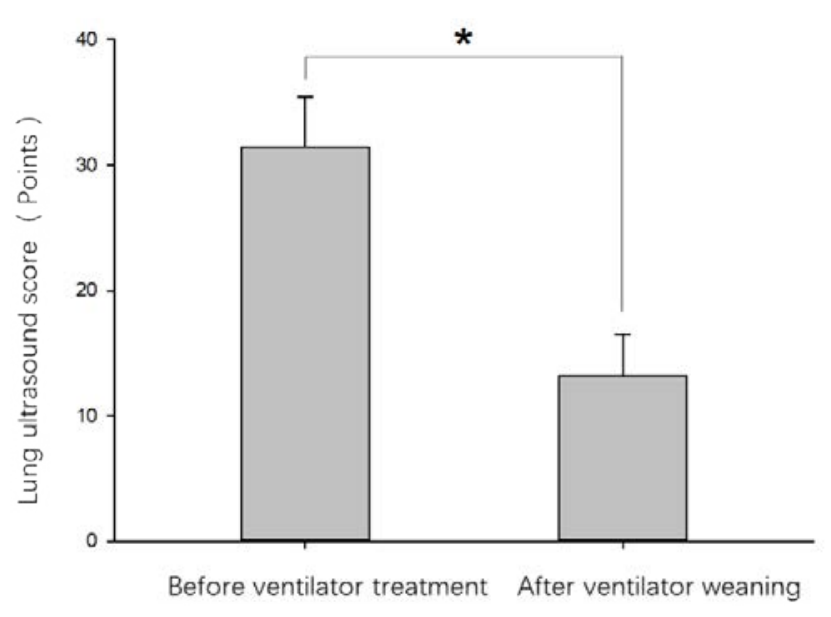

Figure 1. Comparison of lung ultrasound scores before and after ventilator treatment in NRDS neonates
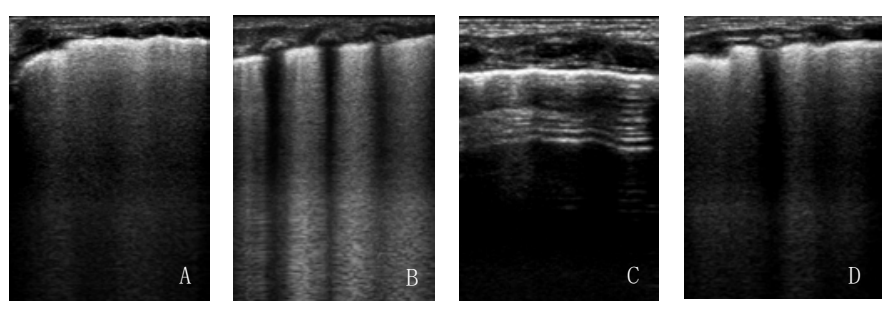

Figure 2. Lung ultrasound imaging findings before and after ventilator treatment in NRDS children (A and B: Lung ultrasonographic manifestations of NRDS patients with combined $\mathrm{b}$-line, dense b-line and white lung before ventilator assisted ventilation; $\mathrm{C}$ : After ventilator assisted ventilation, the symptoms and signs of NRDS children were improved. After removal of the machine, the lung ultrasound showed clear A line, and occasionally isolated $\mathrm{B}$ line. D: Two cases failed to withdraw aircraft for the first time, lung ultrasonographic manifestations deteriorated).

ultrasound could reflect the severity of NRDS before treatment [22]. $\mathrm{Yu}$ found that the sensitivity and specificity of using lung ultrasound score 22.5 to predict the application of invasive mechanical ventilation was $86 \%$ and $64.5 \%$ [23]. In this study, it was found that the changes of lung ultrasound imaging before and after mechanical ventilation were significant and consistent with clinical symptoms and signs. There were significant differences in lung ultrasound scores before and after treatment. Therefore, lung ultrasound and ultrasound score may be used to guide the treatment and efficacy evaluation of mechanical ventilation.

Of course, two of the cases in this study failed at the first disconnection. The imaging findings of lung ultrasound during mechanical ventilation were improved, and the clinical symptoms and signs were improved, suggesting that we could try to withdraw the machine. Unfortunately, respiratory distress reappeared within 12 hours of departure, with worsening sonographic findings of the lungs. This raises the question: Can pulmonary ultrasound predict the success rate of withdrawal? The answer is obviously uncertain. The results of this study showed that the lung ultrasound manifestations and ultrasound scores were synchronized with the symptoms and signs, but there was no evidence to suggest the success rate of withdrawal, which might be related to the complications of NRDS and whether there was oxygen dependence. However, the ultrasonic characteristics of NRDS complications, such as bronchopulmonary dysplasia, have not been unified, which is also a subject that needs further study in the future [24-26].

Lung ultrasound is non-invasive, radiation-free, inexpensive, simple, bedside, readily available for monitoring, and suitable for dynamic observation. At present, it has attracted more and more attention. San Antonio hospital in Italy has even replaced X-ray with pulmonary ultrasound as the preferred diagnostic tool for neonatal pulmonary diseases [15]. Lung ultrasound is easy to learn [27,28]. However, in order to further improve the accuracy of ultrasonic results, lung ultrasound examiners still need to receive standardized training, including neonatologists or sonographers. With the continuous study of lung ultrasound, lung ultrasound is not only used in paediatric medicine, but also in paediatric cardiothoracic surgery. It is used to diagnose pulmonary complications after cardiac surgery [29,30]. Some studies even use lung ultrasound score to evaluate the prognosis after cardiac surgery [31]. This shows that lung ultrasound will have a longerterm future. In conclusion, lung ultrasound has certain application value and specific advantages in guiding NRDS mechanical ventilation treatment. A comprehensive understanding of lung ultrasound and further standardization of its operation and evaluation criteria will enable its further development and popularization. 


\section{References}

1. Shaomei X, Hongmao Y, Xiaoshan Q (2011) Practice of neonatology.

2. Sweet DG, Carnielli V, Greisen G (2017) European Consensus Guidelines on the Management of Respiratory Distress Syndrome - 2016 Update. Neonatology 111: 107125. [Crossref]

3. Zhonghao Z, Hong C (2010) Diagnosis and treatment of respiratory distress syndrome in premature infants. Chinese Journal of Medicine 45: 18-21.

4. Barillari A, Fioretti M (2010) Lung ultrasound: a new tool for the emergency physician. Intern Emerg Med 5: 335-340. [Crossref]

5. Caiulo VA, Gargani L, Caiulo S, Fisicaro A, Moramarco F (2011) Lung ultrasound in bronchiolitis: comparison with chest X-ray. Eur J Pediatr 170: 1427-1433. [Crossref]

6. Colmenero M, Garcia-Delgado M, Navarrete I, López-Milena G (2010) Utility of the lung ultrasound in the intensive medicine unit. Med Intensiva 34: 620-628. [Crossref]

7. Dexheimer Neto FL, Dalcin Pde T, Teixeira C, Beltrami FG (2012) Lung ultrasound in critically ill patients: a new diagnostic tool. J Bras Pneumol 38: 246-256. [Crossref]

8. Elia F, Verhovez A, Molino P, Ferrari G, Aprà F (2011) Lung ultrasound in the reexpansion of pulmonary atelectasis. Intern Emerg Med 6: 461-463. [Crossref]

9. Reissig A, Gramegna A, Aliberti S (2012) The role of lung ultrasound in the diagnosis and follow-up of community-acquired pneumonia. Eur J Intern Med 23: 391-397. [Crossref]

10. Santuz P, Bonetti P, Serra A, Biban P (2010) Ultrasound-guided lung recruitment in a young infant with ARDS. Paediatr Anaesth 20: 895-896. [Crossref]

11. Zechner PM, Seibel A, Aichinger G, Steigerwald M, Dorr K, et al. (2012) [Lung ultrasound in acute and critical care medicine]. Anaesthesist 61: 608-617. [Crossref]

12. Caiulo VA, Gargani L, Caiulo S (2013) Lung ultrasound characteristics of communityacquired pneumonia in hospitalized children. Pediatr Pulmonol 48: 280-287. [Crossref]

13. Copetti R, Cattarossi L, Macagno F (2008) Lung ultrasound in respiratory distress syndrome: a useful tool for early diagnosis. Neonatology 94: 52-59. [Crossref]

14. Elia F, Ferrari G, Molino P, Violino M, Furlan R (2010) Lung ultrasound in postprocedural pneumothorax. Acad Emerg Med 17: e81-82. [Crossref]

15. Cattarossi L, Copetti R, Poskurica B (2011) Radiation exposure early in life can be reduced by lung ultrasound. Chest 139: 730-731. [Crossref]

16. Jing L (2013) Feasibility and Necessity of Pulmonary Ultrasound in Neonatal Intensive Care Unit. J Perinat Med 16: 582-584.

17. Jing L, Junjin H, Ying C (2014) Using lung ultrasound to difference the pulmonary reasons of long-term oxygen dependence in premature infants. Pediatr Em Med 21: 786-789.
18. Jing L (2014) Diagnosis of neonatal respiratory distress syndrome using lung ultrasonography. Journal of Applied Clinical Pediatrics 29: 1438-1440.

19. Jing L (2018) Guideline on lung ultrasound to diagnose pulmonary diseases in newborn infants. Zhongguo Dang Dai Er Ke Za Zh 33: 1057-1064. [Crossref]

20. Bouhemad B, Brisson H, Le-Guen M, Arbelot C, Lu Q, et al. (2011) Bedside ultrasound assessment of positive end-expiratory pressure-induced lung recruitment. Am J Respir Crit Care Med 183: 341-347. [Crossref]

21. Bouhemad B, Liu ZH, Arbelot C, Zhang M, Ferarri F, et al. (2010) Ultrasound assessment of antibiotic-induced pulmonary reaeration in ventilator-associated pneumonia. Crit Care Med 38: 84-92. [Crossref]

22. Xiaohua W, Jian L, Guomei J (2017) Value of bed side lung ultrasound in evaluating neonatal hyaline membrane disease. Journal of Medical Imaging 3: 458-460.

23. Yu Hongkui, Xia Bei, Huang Huijun (2017) Feasibility and clinical significance of lung ultrasound score in assessment on pulmonary lesions and prognosis in neonates with respiratory distress syndrome. Chinese Journal of Medical Imaging Technology 33: 1216-1220.

24. Chen SW, Fu W, Liu J (2017) Routine application of lung ultrasonography in the neonatal intensive care unit. Medicine (Baltimore) 96: e5826. [Crossref]

25. Chen SW, Zhang MY, Liu J (2015) Application of Lung Ultrasonography in the Diagnosis of Childhood Lung Diseases. Chin Med J (Engl) 128: 2672-2678. [Crossref]

26. Liu J, Chen SW, Liu F, et al (2014) BPD, Not BPD, or iatrogenic BPD: findings of lung ultrasound examinations. Medicine (Baltimore) 93: e133. [Crossref]

27. Bedetti G, Gargani L, Corbisiero A, et al (2006) Evaluation of ultrasound lung comets by hand-held echocardiography. Cardiovasc Ultrasound 4: 34. [Crossref]

28. Brusa G, Savoia M, Vergine M, Frassi F, Poggianti E, et al. (2015) Neonatal Lung Sonography: Interobserver Agreement Between Physician Interpreters with Varying Levels of Experience. J Ultrasound Med 34: 1549-1554.

29. Cantinotti M, Giordano R, Valverde I (2017) Lung ultrasound: A new basic, easy, multifunction imaging diagnostic tool in children undergoing pediatric cardiac surgery. J Thorac Dis 9: 1396-1399. [Crossref]

30. Cantinotti M, Ait Ali L, Scalese M, Giordano R, Melo M, et al. (2018) Lung ultrasound reclassification of chest X-ray data after pediatric cardiac surgery. Paediatr Anaesth 28: 421-427. [Crossref]

31. Cantinotti M, Giordano R, Scalese M, Marchese P, Franchi E, et al. (2019) Prognostic value of a new lung ultrasound score to predict intensive care unit stay in pediatric cardiac surgery. Ann Thorac Surg pii: S0003-4975: 31105-1. [Crossref]

Copyright: (C2019 Chun Z. This is an open-access article distributed under the terms of the Creative Commons Attribution License, which permits unrestricted use, distribution, and reproduction in any medium, provided the original author and source are credited. 